\title{
JPEG 2000 CODING OF COLOR-QUANTIZED IMAGES
}

\author{
Armando J. Pinho and António J. R. Neves \\ Dep. Electrónica e Telecomunicações / IEETA \\ U niversidade de Aveiro, 3810-193 Aveiro, Portugal \\ ap@det.ua.pt - an@ieeta.pt
}

\begin{abstract}
The efficiency of compressing color-quantized images using general purpose lossl ess image coding methods depends on the degree of smoothness of the index images. A wellknown and very effective approach for increasing smoothness relies on palette reordering techniques. In this paper, we show that these reordering methods may leave some room for further improvements in the compression performance. More precisely, we provide experimental results showing that the JPEG 2000 lossless compression of pal ette reordered color-quantized natural images can be further improved if histogram packing is applied on a regional basis.
\end{abstract}

\section{INTRODUCTION}

Due to transmission, storage or, most often, display restrictions, there is sometimes a need to keep the number of different colors of an image under a certain limit. In the case of computer-generated images, this limitation is generally imposed during image creation. However, natural images are frequently acquired with a high number of different colors and, therefore, require a color reduction step. To perform this step, known as "color quantization", a number of techniques have been proposed (see, for example, [1, 2, 3, $4,5,6])$. Color quantization artifacts, such as color contouring, can be attenuated by the means of dithering techniques $[7,8,9]$.

Color-quantized images are usually represented by a matrix of indexes (to which we refer to as the "index image") and by a table of colors that establishes the correspondence between indexes and RGB triplets (the pal ette or color map).

In principle, the index images can be compressed by general purpose lossless image coding techniques, al though attaining a coding efficiency that varies according to the particular mapping that is implemented by the color pal ette. In fact, the general purpose image coding techniques, such as JPEG [10], JPEG-LS [11, 12] or JPEG 2000 [13, 14], are based on coding models tuned for images that are smooth

This work was supported in part by the Fundação para a Ciência e a Tecnologia (FCT). almost everywhere. Unfortunately, this assumption is not valid for the generality of the index images resulting from color quantization processes. As a direct consequence, compression performance is usually severely impaired.

In order to minimize this drawback, several techniques have been proposed with the aim of reordering the color map and better fitting the coding model. However, this is not a trivial task, due to the combinatorial nature of the problem [15]. Several sub-optimal solutions have been proposed, which are based on approximated solutions to the traveling salesman problem [16, 17], on the maximization of the compression performance through a greedy index assignment [18], on greedy pairwise merging heuristics [15], or on color reordering by luminance [19].

The pal ette reordering preprocessing techniques improve compression efficiency, sometimes dramatically. N evertheless, we show in this paper that there is still some room for further improvement.

\section{HISTOGRAM PACKING}

Recent work $[20,21]$ has shown that histogram packing is capable of boosting the compression performance of standard lossless image coding techniques, such as JPEG-LS or Iossless J PEG 2000. However, to be able to benefit from this technique an image has to possess a sparse histogram. This is a very common characteristic in gray-scale computer-generated or compound images, but is nonexistent in colorquantized images, at least from a global point of view.

In this paper, we show that histogram packing can also be successfully applied on a local basis, i.e., on regions of the image, even when the image has a globally dense histogram. Figure 1 illustrates this point, presenting the (dense) histogram of a color-quantized image and the (sparse) histogram of a $32 \times 32$ block of the same image.

Histogram packing is a preprocessing technique that can be obtained through the construction of an one-to-one orderpreserving mapping, $h$, from the index values, $\mathcal{I}$, into a contiguous subset of $\mathbb{N}_{0}$ :

$$
h=\left(I_{0} \mapsto 0, I_{1} \mapsto 1, \ldots, I_{N-1} \mapsto N-1\right),
$$




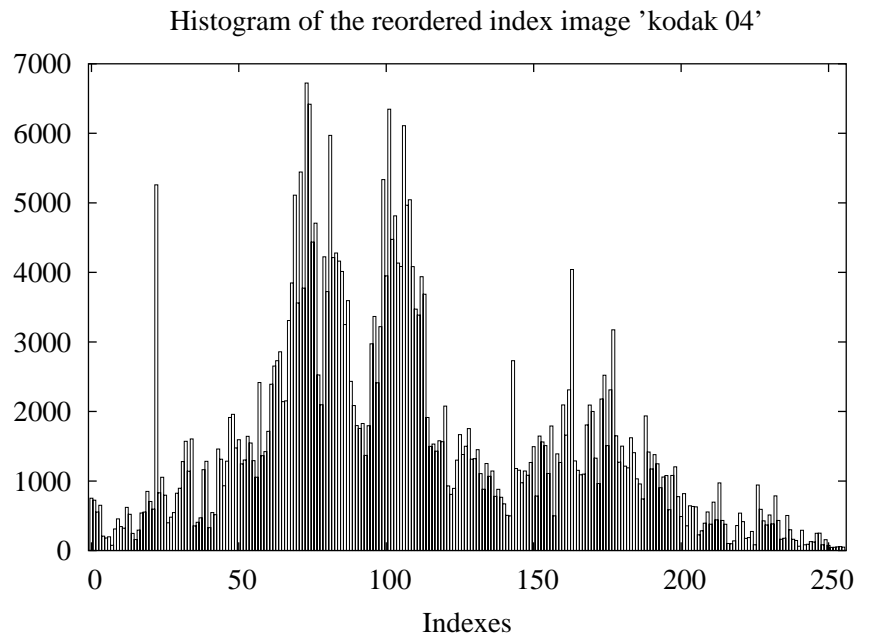

Histogram of the 1 st block of the reordered index image 'kodak 04'

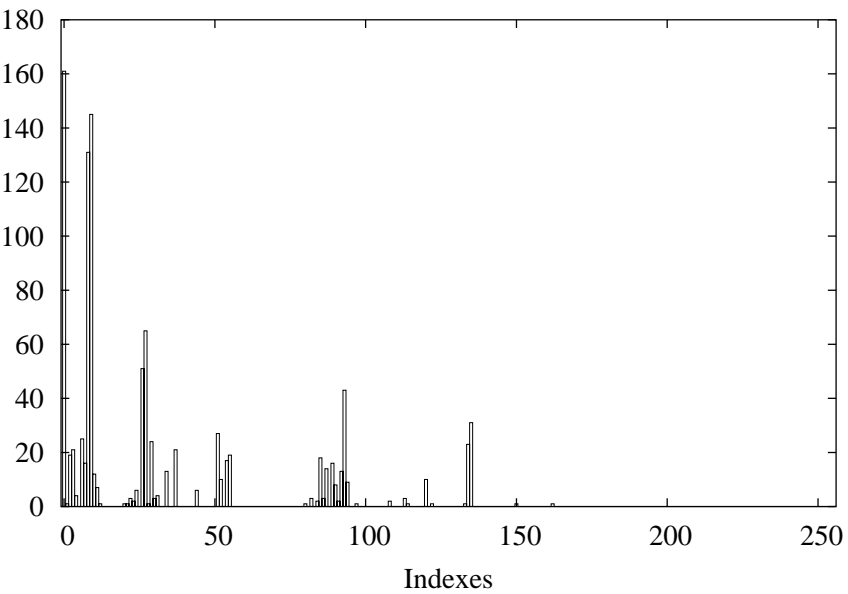

Fig. 1. On the left, the histogram of the reordered "kodak 04 " index image. As can be observed, this histogram is dense, making global histogram packing useless. On the right, we present the histogram of a $32 \times 32$ block of the same image, which is clearly sparse. Therefore, histogram packing can be applied in this case. In this paper, we show that this approach can bring additional compression improvements of the index images.

where $I_{i} \in \mathcal{I}$ and where we assume, without loss of generality, that $I_{i}<I_{j}, \forall_{i<j}$. A fter constructing and applying this mapping to an image region, $r$, a new region $r_{p}=h(r)$ is generated. Compression gains can be obtained if the storage required by the compressed version of $r_{p}$ together with the overhead needed by the recovering process is smaller than the storage required by the compressed version of $r$.

Generally, constructing and applying $h$ implies two passes: the first one to determine $\mathcal{I}$ and the second to apply the mapping $h$. Nevertheless, online strategies have been proposed, some of them designed specifically for a particular encoding al gorithm [22, 23, 24], others designed to work as an independent preprocessing stage [20]. A comparison of some of these methods is presented in [25].

As we mentioned above, in this paper we exploit the idea of local histogram packing, which implies the partition of the image into a set of disjoint regions. The choice of a particular partition has implications on the overall performance of the method, i.e., on the final compression rate. However, it is clearly impractical to search for the best partition among all possible partitions. In this work we rely on the simple case of fixed-shape fixed-size regions and, particularly, on $32 \times 32$ square regions. Figure 2 depicts how the proposed method works.

\section{EXPERIMENTAL RESULTS}

In this section we present experimental results based on the set of the 23 "kodak" color images ${ }^{1}$. These are $768 \times 512$

\footnotetext{
${ }^{1}$ These images can be obtained from ftp://ftp.ipl.rpi.edu/ stills/kodak/color.
}

true color images from which we generated additional sets with resolutions $384 \times 256$ and $192 \times 128$. Color quantization was then applied, both with and without Floyd-Steinberg color dithering, creating images with 256, 128 and 64 colors. Image manipulations have been performed using version 1.2.3 of the "Gimp" program. ${ }^{2}$

Table 1 shows J PEG 2000 lossless compression ${ }^{3}$ results of the reordered index images ${ }^{4}$, with the use of local histogram packing ("Packing" column) and without it ("Normal" column). For reference, we also include compression results using the well-known GIF image file format ${ }^{5}$. Besides the size of the encoded index image, the (uncompressed) size of the color table and the overhead needed for storing the (uncompressed) lists of indexes are also accounted in the results shown in Table 1.

Each row of Table 1 displays the average compression value, in bits per pixel, of a particular instance of the 23 "kodak" images. Therefore, this table incorporates results from a total of 414 different images. A s can be observed, the local histogram packing provides improvements in all presented cases. It can also be noticed that the compression gain increases as the image size and the number of colors increase. M oreover, for the same size and number of colors, images with dithering have a trend to gain more with the proposed technique than color-quantized images without dithering.

\footnotetext{
2http://www.gimp.org.

${ }^{3}$ Compression was obtained using the J asPer 1.700.2 J PEG 2000 codec with default parameters (http://www.ece.uvic.ca/ mdadams/ jasper).

${ }^{4}$ We chose the technique proposed by Zeng et al. for palette reordering, due to its adoption in the JPEG 2000 verifi cation model software [18]

${ }^{5}$ http: //pds-geophys.wustl.edu/info/gif.txt.
} 


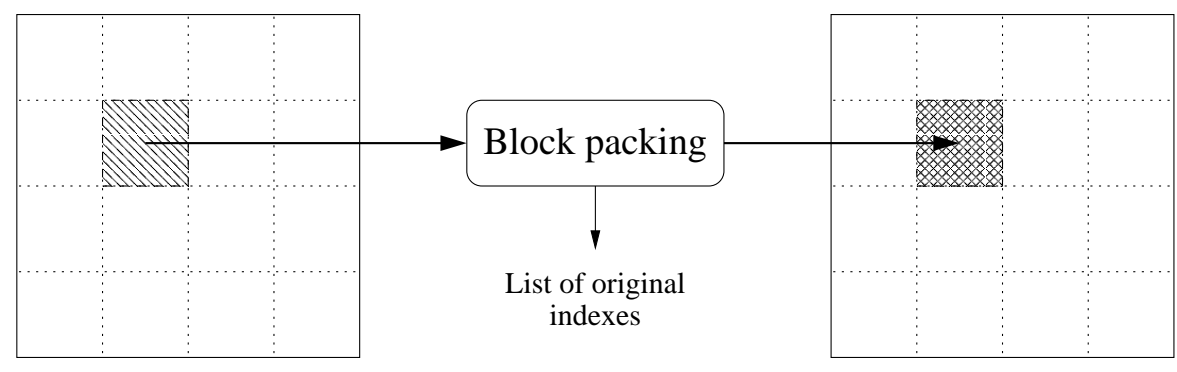

Palette-reordered image

Palette-reordered and block histogram-packed image

Fig. 2. Schematic overview of the proposed local histogram packing method. The overhead data consists of the list of original image indexes that are found in each histogram packed block.

\section{CONCLUSIONS}

Color pal ette reordering is a very effective approach for improving the compression of color-quantized images, even when sub-optimal solutions are considered, having the additional advantages of not implying overheads nor requiring a reverse post-processing phase. N otwithstanding, these methods leave some room for further improvements in the compression performance of the images.

In this paper, we provided experimental results showing that the J PEG 2000 lossless compression of color-quantized natural images can be further improved if histogram packing is applied on a regional basis. In the specific case presented here, we used $32 \times 32$ square regions. However, other shapes and sizes could be used. M oreover, the packing procedure was applied to all regions of all images in a "blind" manner. In a near future, we plan to study the effect of adding a decision mechanism with the aim of only triggering histogram packing when it is advantageous for the image region is question.

\section{ACKNOWLEDGEMENT}

The authors would like to thank Dr. Wenjun Zeng, for providing us the source code of the reordering technique described in [18].

\section{REFERENCES}

[1] M. T. Orchard and C. A. Bouman. Color quantization of images. IEEE Trans. on Signal Processing, 39(12):26772690, December 1991.

[2] S.-C. Pei and Y.-S. L O. Color image compression and limited display using self-organization Kohonen map. IEEE Trans. on Circuits and Systems for Video Technology, 8(2):191205, A pril 1998.
[3] J. Puzicha, M. Held, J. K etterer, J. M. B uhmann, and D. W. Fellner. On spatial quantization of color images. IEEE Trans. on Image Processing, 9(4):666-682, A pril 2000.

[4] A. Mojsilović and E. Soljanin. Color quantization and processing by Fibonacci lattices. IEEE Trans. on Image Processing, 10(10):1712-1725, N ovember 2001.

[5] D. Özdemir and L. A karun. Fuzzy algorithms for combined quantization and dithering. IEEE Trans. on Image Processing, 10(6):923-931, June 2001.

[6] N. Papamarkos, A. E. A tsalakis, and C. P. Strouthopoulos. A daptive color reduction. IEEE Trans. on Systems, Man, and Cybernetics - Part B: Cybernetics, 32(1):44-56, February 2002.

[7] R. Floyd and L. Steinberg. A n adaptive algorithm for spatial grayscale. Proc. Soc. Image Display, 17(2):75-77, 1976.

[8] L. A karun, Y. Yardımcı, and A. E. Çetin. A daptive methods for dithering color images. IEEE Trans. on Image Processing, 6(7):950-955, July 1997.

[9] D. L. Lau, G. R. A rce, and N. C. Gallagher. Digital color halftoning with generalized error diffusion and multichannel green-noise masks. IEEE Trans. on Image Processing, 9(5):923-935, M ay 2000.

[10] W. B. Pennebaker and J. L. M itchell. JPEG still image data compression standard. Van N ostrand Reinhold, N ew York, 1993.

[11] Information technology - Lossless and near-lossless compression of continuous-tone still images. ISO/IEC 14495-1 and ITU Recommendation T.87, 1999.

[12] M. J. Weinberger, G. Seroussi, and G. Sapiro. The L OCO-I lossless image compression algorithm: principles and standardization into J PEG-LS. IEEE Trans. on Image Processing, 9(8):1309-1324, A ugust 2000.

[13] Information technology - JPEG 2000 image coding system. ISO/IEC International Standard 15444-1, ITU-T Recommendation T.800, 2000. 


\begin{tabular}{c|c|c||c||c|c|c}
\hline \hline Image size & Colors & \multicolumn{1}{|c||}{ Dither } & \multicolumn{1}{c||}{ GIF } & \multicolumn{2}{c|}{ Zeng +JPEG 2000 } & Packing \\
\cline { 5 - 6 } & & & & Normal & Packing & gain \\
\hline \hline $192 \times 128$ & 64 & No & 3.965 & 3.819 & 3.730 & $2.3 \%$ \\
& 64 & Yes & 4.371 & 4.242 & 4.136 & $2.5 \%$ \\
& 128 & No & 5.100 & 4.864 & 4.697 & $3.4 \%$ \\
& 128 & Yes & 5.565 & 5.314 & 5.102 & $4.0 \%$ \\
& 256 & No & 6.402 & 6.138 & 5.857 & $4.6 \%$ \\
& 256 & Yes & 6.880 & 6.491 & 6.197 & $4.5 \%$ \\
\hline $384 \times 256$ & 64 & No & 3.498 & 3.389 & 3.282 & $3.2 \%$ \\
& 64 & Yes & 3.924 & 3.934 & 3.739 & $5.0 \%$ \\
& 128 & No & 4.528 & 4.422 & 4.184 & $5.4 \%$ \\
& 128 & Yes & 4.994 & 4.955 & 4.624 & $6.7 \%$ \\
& 256 & No & 5.695 & 5.574 & 5.207 & $6.6 \%$ \\
& 256 & Yes & 6.194 & 6.021 & 5.573 & $7.4 \%$ \\
\hline $768 \times 512$ & 64 & No & 3.270 & 3.147 & 3.009 & $4.4 \%$ \\
& 64 & Yes & 3.730 & 3.808 & 3.536 & $7.1 \%$ \\
& 128 & No & 4.277 & 4.203 & 3.895 & $7.3 \%$ \\
& 128 & Yes & 4.746 & 4.755 & 4.321 & $9.1 \%$ \\
& 256 & No & 5.386 & 5.281 & 4.803 & $9.1 \%$ \\
& 256 & Yes & 5.941 & 5.835 & 5.241 & $10.2 \%$ \\
\hline
\end{tabular}

Table 1. Each row of the table shows average compression results, in bits per pixel, of a particular instance of the "kodak" set of images. The indicated packing gains were calculated in relation to the "Normal" J PEG 2000 lossless compression of the reordered index images. Color table sizes as well as the overheads generated by the histogram packing procedure are included in the compression results.

[14] A. Skodras, C. Christopoulos, and T. Ebrahimi. The JPEG 2000 still image compression standard. IEEE Signal Processing Magazine, 18(5):36-58, September 2001.

[15] N. D. Memon and A. Venkateswaran. On ordering color maps for lossless predictive coding. IEEE Trans. on Image Processing, 5(5):1522-1527, N ovember 1996.

[16] S. B attiato, G. Gallo, G. Impoco, and F. Stanco. A color reindexing al gorithm for lossless compression of digital images. In Proc. of the IEEE Spring Conf. on Computer Graphics, pages 104-108, Budmerice, Slovakia, A pril 2001.

[17] A. Spira and D. M alah. Improved lossless compression of color-mapped images by an approximate solution to the traveling salesman problem. In Proc. of the IEEE Int. Conf. on Acoustics, Speech, and Signal Processing, ICASSP-2001, pages 1797-1800, Salt Lake City, UT, M ay 2001.

[18] W. Zeng, J. Li, and S. Lei. An effi cient color re-indexing scheme for palette-based compression. In Proc. of the 7th IEEE Int. Conf. on Image Processing, ICIP-2000, pages 476-479, Vancouver, Canada, September 2000.

[19] A. Zaccarin and B. Liu. A novel approach for coding color quantized images. IEEE Trans. on Image Processing, 2(4):442-453, October 1993.
[20] A. J. Pinho. An online preprocessing technique for improving the lossless compression of images with sparse histograms. IEEE Signal Processing Letters, 9(1):5-7, J anuary 2002.

[21] P. J. S. G. Ferreira and A. J. Pinho. Why does histogram packing improve lossless compression rates? IEEE Signal Processing Letters, 9(8):259-261, A ugust 2002.

[22] R. Ansari and N. M emon. The JPEG lossless compression standards. In A. B ovik, ed., Handbook of image and video processing, A cademic Press, 2000.

[23] Information technology - Lossless and near-lossless compression of continuous-tone still images: extensions. ISO/IEC 14495-2, 2000.

[24] A. J. Pinho. On the impact of histogram sparseness on some lossless image compression techniques. In Proc. of the 8th IEEE Int. Conf. on Image Processing, ICIP-2001, pages 442-445, Thessaloniki, Greece, 0 ctober 2001.

[25] A. J. Pinho. A comparison of methods for improving the lossless compression of images with sparse histograms. In Proc. of the 9th IEEE Int. Conf. on Image Processing, ICIP2002, pages 673-676, R ochester, NY, September 2002. 